\title{
Benoît-Michel Tock
}

\section{OpenEdition \\ Journals}

Édition électronique

URL : http://journals.openedition.org/ifha/1740

DOI : $10.4000 /$ ifha. 1740

ISSN : 2198-8943

\section{Éditeur}

IFRA - Institut franco-allemand (sciences historiques et sociales)

\section{Référence électronique}

Benoît-Michel Tock, « », Revue de I'IFHA [En ligne], Date de recension, mis en ligne le 01 janvier 2009, consulté le 22 septembre 2020. URL : http://journals.openedition.org/ifha/1740 ; DOI : https://doi.org/ 10.4000/ifha. 1740

Ce document a été généré automatiquement le 22 septembre 2020.

(C)IFHA 
Benoît-Michel Tock

1 Si le catalogage de manuscrits est une pratique ancienne, plongeant ses racines au Moyen Âge même, cette branche de l'érudition a connu des développements inégaux selon les pays. Fière de la tradition allemande en la matière, la Bayerische Staatsbibliothek a organisé en 2005 un colloque destiné à faire le point dans un cadre international sur les pratiques actuelles de catalogage. Les 22 articles qui en ont résulté sont répartis dans six sections, qui cependant ne s'imposent pas vraiment. On peut surtout faire la différence entre des articles faisant l'histoire du catalogage et d'autres qui présentent des réalisations sur internet.

2 La mise au point, dans les années 1960 en Allemagne par exemple, de normes modernes de description des manuscrits a imposé des descriptions beaucoup plus précises, aussi bien du point de vue codicologique que textuel, ce qui a fait faire de très considérables progrès à l'histoire de la tradition d'un texte comme à l'identification de textes peu connus, et a également permis de trouver des textes inconnus, comme cette collection de 30 lettres et mandements de l'empereur Frédéric II et de plus de 100 lettres de son fils Conrad IV, trouvée dans un manuscrit rapidement inventorié comme des notulae rhetoricales diversae (Innsbruck, Universitätsbibl., cod. 400). C'est F. FUCHS qui relate cette découverte, ainsi que quelques autres, tandis que M. C. FERRARI retrace l'histoire du catalogage des manuscrits et vante les progrès ainsi réalisés ; R. SHARPE, lui, montre par deux exemples concrets comment les catalogues de manuscrits peuvent aider à identifier des textes médiolatins. G. Z. ZANICHELLI raconte l'histoire du catalogage des manuscrits enluminés de Parme. A. FINGERNAGEL insiste sur la nécessité, quand on rédige un catalogue de manuscrits, de décrire aussi la décoration des codices. La description, extrêmement précise, doit porter sur l'ensemble du volume et utiliser le vocabulaire technique. Une reproduction au moins par manuscrit lui paraît un minimum. En complément, U. BAUER-EBERHARDT, historienne de l'art en charge des quelque 500 manuscrits italiens enluminés de la Bayerische Staatsbibliothek, explique que les bases de données ne lui sont guère utiles et qu'elle utilise encore surtout les méthodes traditionnelles de l'histoire de l'art. Les exigences d'A. FINGERNAGEL ne pouvant être réalisées en un jour, M. REUTER propose un programme progressif, en quatre étapes, pour aboutir non seulement au catalogue, mais aussi à la mise en ligne au moins d'extraits significatifs, sinon de la totalité, de quelque 130 codices iconographici de Munich. C. GLASER ne partage cependant pas leur optimisme. La 
comptabilité qu'elle tient est d'ailleurs impitoyable : sur les 25276 codices appartenant aux 19 bibliothèques autrichiennes riches de plus de 250 manuscrits, seuls 5220 ont bénéficié de notices dans des catalogues postérieurs à 1940. La bibliothèque universitaire de Graz et celle de l'abbaye de Zwettl sont les seules à avoir inventorié la totalité de leurs manuscrits médiévaux (au nombre de 1217 dans le premier cas, de 424 dans le second). Pour remédier à cette situation, C. GLASER propose de mettre au point un catalogage par étapes, afin de publier d'abord pour tous les manuscrits les informations les plus importantes, pour revenir ensuite sur d'autres informations.

3 Les réalisations sur internet sont nombreuses. F. QUEYROUX présente le Consortium of European Research Libraries, qui donne accès à une base de données de références de livres imprimés avant 1830, mais également à un Thesaurus qui est un vaste lexique multilingue des noms de personnes et de lieux trouvés dans les livres de cette même période. Et qui surtout a construit un portail donnant accès aux catalogues de plusieurs bibliothèques. A.-M. HANSEN explique comment un projet dano-islandais va permettre la réunification virtuelle, par le biais d'un unique catalogue, de la collection " Arnamagnæn ", constituée par Árni Magnússon, un érudit islandais et professeur à l'université de Copenhague (1663-1730) et divisée entre Islande et Danemark aux termes d'un accord conclu en 1971. Autres bases de données : le « Digital Catalogue of Illuminated Manuscripts » de la British Library, mis en valeur par M. HOFMAN ; la collection de manuscrits mérovingiens et carolingiens de la Bibliothèque Municipale de Lyon, présentée par M. HULVEY.

4 Il y a quelques autres interventions. K.-H. KELLER rappelle qu'il faut considérer la bibliothèque non comme un ensemble de manuscrits séparés, mais comme un ensemble à étudier en lui-même, constitué progressivement, et caractérisé non seulement par un choix de manuscrits, mais aussi par des regroupements, des annotations... Pour D. STUTZMANN et P. TYLUS, la coopération internationale doit caractériser toute étude de manuscrits, dans la mesure où les scribes d'abord, leurs manuscrits ensuite, ont beaucoup voyagé. Ils en prennent pour exemple le fonds de manuscrits français de la Preussische Staatsbibliothek transférés de Berlin à Cracovie. A. VIZKELETY présente les catalogues de manuscrits médiévaux dans les bibliothèques hongroises, J. BALAZS VEBER les manuscrits médiévaux de la bibliothèque de l'Académie Hongroise des Sciences, M. PORIEZOVA les manuscrits de la Bibliothèque Universitaire de Bratislava, et $\mathrm{E}$. MOUSSAKOVA s'interroge sur la terminologie utilisée pour décrire les manuscrits enluminés slavons.

5 Enfin, la Bayerische Staatsbibliothek profite de l'occasion pour mettre en avant ses richesses. B. GULLATH signale les ressources qu'offre cette bibliothèque pour aider à la description de manuscrits. H. HAUKE retrace l'histoire du catalogage de manuscrits en Bavière ; son article est suivi par une bibliographie des catalogues de manuscrits occidentaux de la Bayerische Staatsbibliothek.

6 Dans l'ensemble, le catalogage des manuscrits, comme bien d'autres branches de l'érudition en médiévistique, est un peu à la croisée des chemins : le bilan est impressionnant, mais la qualité a nettement pris le pas sur la quantité, laissant de côté de très nombreux manuscrits. Les catalogues mis en ligne sur internet marquent un progrès, mais ils n'interfèrent pas dans cette question décisive, et si difficile à trancher : faut-il mettre l'accent sur la quantité ou sur la qualité ? En l'occurrence, c'est la méthode proposée par C. GLASER qui me paraît la plus équilibrée.

7 Benoît-Michel TOCK (Université Marc-Bloc Strasbourg II) 